\title{
AVALIAÇÃO DA ASSISTÊNCIA MATERNO-INFANTIL PRESTADA POR UMA EQUIPE RURAL DO PROGRAMA SAÚDE DA FAMÍLIA
}

\author{
Evaluation of the Maternal-Infantile Attendance Give \\ by a Rural Health Team of the Family Program \\ Evaluación de la Atención Materno-Infantil por un \\ Equipo Rural del Programa de Salud de La Familia
}

\author{
Claudete Costa de Lima \\ Ana Augusta Monteiro Cavalcante
}

Rosângela Minardi Mitre Cotta

Poliana Cardoso Martins

\begin{abstract}
Resumo
0 presente estudo visa avaliar a qualidade da assistência à saúde infantil e as estratégias implementadas no serviço de puericultura de uma equipe do Programa de Saúde da Família, do município de Teixeiras-MG, enfocando a situação de saúde e nutrição das famílias estudadas. Investigaram-se os prontuários de todas as puérperas (49) acompanhadas pela assistência pré-natal e selecionaram-se os prontuários (26) dos recém-nascidos que compareceram à consulta do serviço de puericultura. A média de idade materna foi 27,5 anos $( \pm 5,5)$. A maioria das mães $(81,8 \%)$ teve gestação a termo. 0 peso médio das crianças ao nascer foi de 3.060 ( $\pm 448 \mathrm{~g}$ ), 63,6\% compareceram a menos de 4 consultas durante o período estudado, $100 \%$ das crianças eram amamentadas e apenas 8,3\% referiram história de internação hospitalar. Os resultados permitiram a identificcação de importantes informações sobre a qualidade da assistência prestada ao grupo materno-infantil e contribuíram para auxiliar o planejamento de ações efetivas que possibilitaram um melhor conhecimento das condições de saúde.
\end{abstract}

Palavras-chave: Criança. Serviços de Saúde. Programa Saúde da Família.

\begin{abstract}
The present study aims to evaluate the quality of the assistance to the infantile health and the strategies implemented in the child care service of a team of the Family Health Program, of the city of Teixeiras - Minas Gerais (Brazil), focusing the situation of health and nutrition of the studied families. It was investigated the medical records of all puérperas (49) followed by the prenatal assistance and selected medical records (26) of the newborn that had appeared to the consultation of the child care service. The maternal average age was 27,5 years old $( \pm 5,5)$. The majority of the mothers $(81,8 \%)$ had a term gestation. The average weight of the children when born was of 3.060 ( $\pm 448 \mathrm{~g}$ ), $63.6 \% \mathrm{had}$ appeared in less than 4 consultations during the studied period. $100 \%$ of the children were breast fed and only $8.3 \%$ had related history of hospital internment. The results had allowed the identification of important information about the quality of the assistance given to the maternal-infantile group and had contributed to assist the planning of effective actions that made possible a better knowledge of the health conditions.
\end{abstract}

Keywords:

Child. Health Services. Family Health Program.

\section{Resumen}

El estudio tiene como objetivo evaluar la calidad de la atención de la salud de los niños y las estrategias implementadas en el servicio de puericultura de un equipo rural del Programa de Salud de la Familia, de Teixeiras - Minas Gerais (Brasil), con énfasis en la situación de salud y nutrición de las familias estudiadas. Fueron investigados los prontuarios de todas las puérperas (49) de atención prenatal y se realizó la selección de los (26) prontuarios de los recién-nacidos que comparecieron a la consulta del servicio de puericultura. La edad media de las madres fue 27,5 anos $( \pm 5,5)$. La mayoría de las madres $(81,8 \%)$ tuvieron el embarazo en el período normal. El peso medio al nacimiento fue 3.060 ( $\pm 448 \mathrm{~g}$ ), 63,6\% participaron de menos de 4 consultas, $100 \%$ de los niños fueron amamantados y sólo 8,3\% informaron historia de ingreso hospitalario. Los resultados llevaron a la identificación de importantes informaciones sobre la calidad de la atención ofrecida al grupo de las madres y de los niños y contribuyeron para el planeamiento de las acciones efectivas que posibilitaron un mejor conocimiento de las condiciones de salud.
Palabras clave:

Niño. Servicios de Salud. Programa Salud de la Familia. 


\section{INTRODUÇÃO}

0 Programa Saúde da Família (PSF) tem como premissa prestar assistência integral à saúde de grupos populacionais previamente definidos, respondendo de forma contínua e racionalizada às demandas populacionais, visando principalmente promover a saúde por meio da educação sanitária'.

Neste contexto, a família é considerada o núcleo central da atenção à saúde, onde os indivíduos com suas condições de moradia, trabalho, alimentação, educação e lazer, entre outras, interagem com uma equipe de profissionais de saúde, a fim de diagnosticar as situações que afetam o seu bem-estar, compartilhando, assim, as responsabilidades no encaminhamento das soluções para cada caso detectado².

Dentre os grupos-alvo de atenção do PSF, destaca-se o grupo materno-infantil, que enfoca principalmente a necessidade do cuidado e da promoção da saúde à mulher e à criança, partindo do pressuposto de que o desenvolvimento de ações destinadas ao cuidado da saúde das mulheres pode gerar um impacto direto na redução dos índices negativos de saúde da criança, como a pré-maturidade, baixo peso ao nascer, dentre outros ${ }^{3,4}$.

Desta forma, o acompanhamento profissional para esse grupo populacional se justifica pela necessidade de orientação e controle sobre questões relacionadas ao aleitamento materno, crescimento e desenvolvimento, introdução ao esquema de imunização, assistência e controle às doenças prevalentes na infância ${ }^{5}$, principalmente pelo fato de estas ações utilizarem uma metodologia assistencial simples, econômica, não traumática, aceitável culturalmente e que propicia a identificação das crianças com déficit nutricional ou em risco de adoecerem, favorecendo, assim, uma intervenção precoce e eficaz ${ }^{6}$.

Portanto, após a assistência pré-natal, é essencial o acompanhamento da saúde das crianças até os 5 anos de idade, isto é, o acompanhamento de puericultura. Essas ações representam vital importância no acompanhamento do crescimento e desenvolvimento da criança, fase caracterizada prioritariamente por ser um processo intenso no qual a criança está em grande vulnerabilidade, devido ao crescimento rápid $0^{5,7}$.

A avaliação integral da qualidade do serviço prestado na área da assistência materno-infantil, que inclua indicadores de processo, estrutura e resultado, é uma abordagem de fundamental importância para a organização de um sistema de monitoramento de qualidade de rotina deste serviço, possibilitando o melhor acompanhamento destes programas e auxiliando na análise das necessidades e no planejamento das prioridades e intervenções necessárias no setor ${ }^{4}$.

Outro ponto importante é a presença da equipe multidisciplinar no PSF, que contribui para a mudança na forma de atenção ao grupo materno infantil, principalmente por permitir que a práxis da atenção deixe de ser estritamente médica e especializada, passando a ser desenvolvida mais como um processo multiprofissional e de parceria com as famílias e a comunidade 8,9,10 $^{8,}$

Neste sentido, este estudo tem como objetivo avaliar a qualidade da assistência à saúde infantil e as estratégias implementadas no serviço de puericultura de uma equipe do Programa de Saúde da Família, descrevendo o perfil das crianças atendidas no serviço de puericultura de uma unidade rural do PSF do município de Teixeiras-MG, e de suas mães, e discutindo a qualidade da assistência prestada, visando contribuir de forma positiva no desenvolvimento de ações de atenção à saúde da criança no tocante à estratégia implementada após a assistência pré-natal.

\section{MÉTODOS}

Trata-se de um estudo observacional descritivo retrospectivo, no qual foram selecionados inicialmente os dados contidos nos prontuários de todas as puérperas $(n=49)$ acompanhadas no programa de assistência pré-natal, durante o período de abril de 2002 a novembro de 2003, de uma equipe rural do PSF no município de Teixeiras, localizado na Zona da Mata de Minas Gerais. De todas as puérperas, foram selecionados e analisados em profundidade os prontuários dos recém-nascidos que compareceram às consultas de puericultura neste mesmo período $(n=26)$. Assim, temos como critério de inclusão das crianças, no presente estudo, o acompanhamento da mãe durante o pré-natal e comparecimento às consultas da puericultura, sendo estas identificadas por meio dos prontuários do serviço. Ressalta-se que resultados aqui apresentados representam $100 \%$ dos prontuários disponíveis.

Dentre as variáveis contidas nos prontuários do serviço de puericultura, foram selecionadas aquelas consideradas importantes para a avaliação da qualidade da assistência à saúde infantil, ou seja, aquelas relevantes para o diagnóstico e acompanhamento da situação clínico-nutricional.

Neste sentido, as variáveis pesquisadas foram: idade e sexo da criança, número de consultas pré-natais, data de início do pré-natal, presença da mãe na consulta do puerpério, renda familiar, idade e escolaridade dos pais, condições de moradia, composição familiar, abastecimento de água e saneamento, destino dos dejetos e do lixo, características do acesso a bens básicos e de consumo e as condições de nascimento (peso ao nascer, condições de saúde incluindo a freqüência de consultas na puericultura, duração do aleitamento materno, prevalência de aleitamento materno exclusivo, cobertura vacinal e as internações).

Utilizou-se o programa Epi-Info (6.04) para a construção, armazenamento, verificação da consistência e análise dos dados ${ }^{11}$. Para a análise, consideraram-se a freqüência absoluta das ocorrências e o cálculo da Razão de Chance (Odds Ratio) entre as variáveis, utilizando o nível de significância estatístico de $5 \%$ ou $p<0,05$.

Respeitando os aspectos éticos, conforme Resolução 196/ 96 do Conselho Nacional de Saúde, o projeto de pesquisafoi submetido e aprovado pelo Comitê de Ética da Universidade Federal de Viçosa.

\section{RESULTADOS}

\section{Características gerais das crianças atendidas no serviço de puericultura}

Das 49 mães atendidas pela equipe durante o período estudado, apenas $26(53 \%)$ levaram seus filhos à consulta de puericultura, sendo que entre as crianças avaliadas $61,5 \%$ eram do sexo masculino e $38,5 \%$ eram do sexo feminino. Em 
relação à faixa etária na primeira consulta no serviço, observou-se que $80,8 \%$ das crianças compareceram antes dos dois meses de idade.

\section{Características familiares das crianças}

$\mathrm{Na}$ Tabela1 encontram-se as características socioeconômicas das famílias das crianças acompanhadas pela assistência pré-natal e na puericultura. A renda familiar da população em estudo é muito baixa, onde $96,2 \%$ recebem meio ou menos de meio salário mínimo, tendo como base 0 valor de $R \$ 200,00 / R \$ 240,00$, correspondente ao valor dos salários mínimos vigentes no período estudado (abril/2002 a novembro/2003). Observa-se que essas crianças pertencem a grupos familiares em que a renda per capta é inferior a $R \$$ 60,00 , o que implica na baixa disponibilidade de recursos para suprir as necessidades essenciais a uma qualidade digna de vida e acesso mínimo à alimentação, vestuário, habitação, educação, saúde, lazer, etc.

Tabela 1: Caracterização das condições socioeconômicas das crianças atendidas no pré-natal e na puericultura - $2^{a}$ ESF - Zona Rural, Teixeiras - MG, 2002 - 03.

\begin{tabular}{|c|c|c|}
\hline VARIÁVEIS & \multicolumn{2}{|c|}{ FREQÜÊNCIA } \\
\hline RENDA FAMILIAR & $\mathrm{N}$ & $\%$ \\
\hline d"1 salário & 24 & 92,3 \\
\hline$>1$ salário & - & - \\
\hline $\mathrm{SI}{ }^{*}$ & 2 & 7,7 \\
\hline \multicolumn{3}{|l|}{$\begin{array}{l}\text { ESCOLARIDADE } \\
\text { PATERNA }\end{array}$} \\
\hline d"4 anos & 15 & 57,7 \\
\hline$>4$ anos & 5 & 19,2 \\
\hline $\mathrm{SI}{ }^{*}$ & 6 & 23,1 \\
\hline \multicolumn{3}{|l|}{$\begin{array}{c}\text { ESCOLARIDADE } \\
\text { MATERNA }\end{array}$} \\
\hline d"4 anos & 18 & 69,2 \\
\hline$>4$ anos & 5 & 19,3 \\
\hline $\mathrm{SI}{ }^{*}$ & 3 & 11,5 \\
\hline \multicolumn{3}{|l|}{ IDADE PATERNA } \\
\hline$<20$ anos & - & - \\
\hline e"20 anos & 25 & 96,2 \\
\hline $\mathrm{SI}{ }^{*}$ & 1 & 3,9 \\
\hline \multicolumn{3}{|l|}{ IDADE MATERNA } \\
\hline$<20$ anos & 3 & 11,5 \\
\hline e"20 anos & 23 & 88,5 \\
\hline
\end{tabular}

Ainda, conforme se pode visualizar na Tabela 1, no que se refere à escolaridade do pai e da mãe, mais de dois terços das mães avaliadas $(69,2 \%)$ tinham quatro ou menos de quatro anos de estudo (correspondendo ao ensino fundamental incompleto), enquanto apenas 19,2\% estudaram mais de quatro anos. Destacando-se, portanto, que as mães das crianças deste estudo apresentaram baixos níveis educacionais.
Com relação à escolaridade paterna, mais da metade dos pais $(57,7 \%)$ tiveram quatro ou menos anos de estudo, expressando igualmente uma baixa escolaridade.

A média da idade materna foi de 27,5 anos $( \pm 5,5)$, e a mediana, de 27 anos de idade (mínima de 18 e máxima de 38 anos). Não obstante, é importante destacar que $11,5 \%$ das mães estudadas tinham menos de 20 anos de idade e $88,5 \%$ tinham idade igual ou superior a 20 anos (Tabela 1).

As condições de moradia e de saneamento do grupo materno-infantil estão descritas na Tabela 2. Pôde-se observar que $66,8 \%$ das famílias estudadas apresentaram entre dois a quatro habitantes por domicílio. Em relação à avaliação das condições de saneamento, encontrou-se que em aproximadamente $54 \%$ das moradias se utilizava água de mina (água de nascente), sendo que apenas 15,4\% possuíam abastecimento público de água.

Tabela 2: Condições de moradia e de saneamento do grupo materno-infantil - $2^{a}$ ESF - Zona rural, Teixeiras-MG, 2002-2003.

\begin{tabular}{|c|c|c|}
\hline VARIÁVEIS & \multicolumn{2}{|c|}{ FREQÜÊNCIA } \\
\hline$N^{\circ}$ DE MORADORES & $\mathrm{N}$ & $\%$ \\
\hline 2 - 4 moradores & 14 & 53,9 \\
\hline 4 - 5 moradores & 04 & 15,4 \\
\hline 5 ou + & 03 & 11,5 \\
\hline $\mathrm{SI}{ }^{*}$ & 05 & 19,2 \\
\hline \multicolumn{3}{|l|}{$\begin{array}{c}\text { ABASTECIMENTO } \\
\text { DE ÁGUA }\end{array}$} \\
\hline Encanada & 04 & 15,4 \\
\hline Poço & 02 & 7,7 \\
\hline Mina & 14 & 53,9 \\
\hline Outros & 03 & 11,5 \\
\hline $\mathrm{SI} *$ & 03 & 11,5 \\
\hline \multicolumn{3}{|l|}{$\begin{array}{c}\text { DESTINO DOS } \\
\text { DEJETOS HUMANOS }\end{array}$} \\
\hline Fossa & 06 & 23,1 \\
\hline Ribeirão & 09 & 34,6 \\
\hline Esgoto & 02 & 7,7 \\
\hline Outros & 04 & 15,4 \\
\hline $\mathrm{SI}{ }^{*}$ & 05 & 19,2 \\
\hline \multicolumn{3}{|l|}{ DESTINO DO LIXO } \\
\hline Coletado & 05 & 19,2 \\
\hline Queimado & 14 & 53,9 \\
\hline Enterrado & 02 & 7,7 \\
\hline Céu aberto & - & - \\
\hline $\mathrm{SI}{ }^{*}$ & 05 & 19,2 \\
\hline
\end{tabular}

Ainda de acordo com a Tabela 2, quanto ao destino do lixo, verificou-se que em $53,9 \%$ das residências este era queimado. Em relação aos dejetos, observou-se que a maior parte, $(35,0 \%)$ eram despejados diretamente no ribeirão e apenas $8,0 \%$ dos domicílios dispunham de fossa séptica. Estes resultados apontam para uma precariedade das condições de 
saneamento básico nos domicílios, refletindo negativamente nas condições de saúde da população.

Em relação ao acesso aos bens básicos e de consumo, observou-se que as crianças deste estudo pertencem a famílias nas quais $53,8 \%$ possuíam televisão, $38,5 \%$ possuíam geladeira e $87,5 \%$ filtro de água.

\section{Fatores que podem justificar o comparecimento às consultas de puericultura e o impacto desta ação sobre as condições de saúde das crianças}

Foram avaliados fatores de risco que poderiam se correlacionar e justificar o não-comparecimento da criança no serviço de puericultura; para tal realizamos a comparação entre todas as 49 puérperas. Dentre os fatores selecionados, destacamse a idade da mãe, localização do domicílio, o número de consultas e acompanhamento precoce durante o período pré-natal e comparecimento da mãe com o bebê a consulta puerperal.

Os resultados dos cálculos da Razão de Chances (Odds Ratio) entre estas variáveis e a variável consulta da puericultura não se mostraram estatisticamente significantes. Entretanto, se faz importante apresentar estes resultados na forma de freqüência absoluta.

Das mães adolescentes, 75\% levaram seus filhos para a consulta. Dentre as mães de outras faixas etárias, a prevalência observada foi de $54,8 \%$. Com relação às crianças que residiam em comunidades mais acessíveis à Unidade Básica de Saúde, $60,7 \%$ compareceram à consulta, e, em contrapartida, entre as que moravam em locais de difícil acesso, a prevalência foi de 42,9\%. 0 acompanhamento pré-natal pela equipe de saúde da família, principalmente para aquelas mães que realizaram 6 ou mais consultas durante a gestação, pode ser considerado como um fator positivo e motivador para que as mães levem a criança à consulta da puericultura. Neste estudo, observou-se que $67,6 \%$ das mães acompanhadas no pré-natal com estas características levaram suas crianças à consulta da puericultura. 0 período de início do pré-natal também se mostrou importante, visto que $60 \%$ das mães que iniciaram a consulta no primeiro trimestre de gestação compareceram com seus filhos na consulta da puericultura, enquanto no grupo das que iniciaram após este período, a prevalência de comparecimento foi de $48,3 \%$. Tabela 3: Condições de nascimento das crianças atendidas no
serviço de puericultura, filhas das gestantes acompanhadas no
pré-natal pela $2^{a}$ ESF - Zona rural, Teixeiras - MG, 2002- 03 .

\begin{tabular}{c|c|c}
\hline VARIÁVEIS & \multicolumn{2}{|c}{ FREQUÊNCIA } \\
\hline DURAÇÃO & $\mathrm{N}$ & $\%$ \\
DA GESTAÇÃO & & \\
\hline a termo & 18 & 69,2 \\
pré-termo & 3 & 11,5 \\
pós-termo & 1 & 3,9 \\
SI* & 4 & 15,4 \\
\hline PESO AO NASCER & & \\
\hline$<2.500 \mathrm{~g}$ & 02 & 7,7 \\
2.500 $13.000 \mathrm{~g}$ & 10 & 38,5 \\
$>3.000 \mathrm{~g}$ & 14 & 53,8 \\
\hline *Sem informação & \multicolumn{2}{|l}{}
\end{tabular}

A presença da mãe na consulta do puerpério também se mostrou um fator determinante para seguir com 0 acompanhamento da criança. Dentre as mães que foram à consulta, $64,5 \%$ levaram seu filho para o acompanhamento na puericultura, e dentre aquelas que não compareceram à consulta pós-gestacional, apenas 33,3\% levaram as crianças para o atendimento.

Conforme os resultados mostrados na Tabela 3, dentre as mães que levaram seus filhos ao serviço de puericultura, $81,8 \%$ tiveram a gestação a termo, $11,5 \%$ pré-termo e 3,9\% póstermo. A média do peso ao nascer das crianças foi de 3.060 $( \pm 448 \mathrm{~g})$, sendo que $38,5 \%$ nasceram com peso insuficiente e 7,7\% nasceram com baixo peso ao nascer. Estes resultados justificam a importância do acompanhamento pré-natal.

Os dados da Tabela 4 mostram o número de consultas realizadas na puericultura, onde se pode verificar que $63,6 \%$ das crianças compareceram a 4 ou menos consultas e $36,4 \%$ a mais de 4, sendo a mediana de consultas 4,0; o mínimo de comparecimento foi 1 consulta, e o máximo, 16 consultas.

Tabela 4: Indicadores de saúde das crianças atendidas no serviço de puericultura, filhas das gestantes acompanhadas no pré-natal - 2a ESF - Zona rural, Teixeiras - MG, 2002-2003.

\begin{tabular}{|c|c|c|}
\hline VARIÁVEIS & \multicolumn{2}{|c|}{ FREQUÊNCIA } \\
\hline $\begin{array}{l}N^{0} \text { DE CONSULTAS } \\
\text { NO PSF }(N=22)\end{array}$ & $\mathrm{N}$ & $\%$ \\
\hline d"4 consultas & 14 & 63,64 \\
\hline$>4$ consultas & 8 & 36,36 \\
\hline $\begin{array}{c}\text { ALEITAMENTO } \\
\text { MATERNO }(\mathrm{N}=26)\end{array}$ & & \\
\hline Sim & 26 & 100 \\
\hline Não & - & - \\
\hline \multicolumn{3}{|l|}{ VACINAÇÃO (N = 26) } \\
\hline Completa & 22 & 84,62 \\
\hline Incompleta & 1 & 3,84 \\
\hline SI* & 3 & 11,54 \\
\hline \multicolumn{3}{|l|}{ INTERNAÇÕES $(N=26)$} \\
\hline Sim & 2 & 7,69 \\
\hline Não & 22 & 84,62 \\
\hline $\mathrm{SI}{ }^{*}$ & 2 & 7,69 \\
\hline
\end{tabular}

Na Tabela 4 ainda estão destacadas as informações sobre a imunização da população investigada. Dentre as crianças estudadas, apenas 3,8\% estavam com alguma vacina atrasada no momento da coleta dos dados. Entretanto, algumas apresentaram um percentual de ausência de informação $(11,5 \%)$ considerado relevante.

As informações obtidas sobre o aleitamento materno mostraram que todas as crianças estudadas receberam o leite materno. Diante da recomendação da Organização Mundial da Saúde ${ }^{12}$ para a duração do aleitamento exclusivo ( 6 meses) e a do aleitamento total ( 0 - 24 meses ou mais), encontrou-se período mediano de aleitamento materno exclusivo de 
4,48 meses e 7,25 meses de aleitamento total. No que se refere às internações hospitalares, $8,3 \%$ das crianças estudadas já sofreram internação hospitalar.

\section{DISCUSSÃO}

Em relação às características biológicas das crianças atendidas no Serviço de Puericultura do PSF de Teixeiras, MG, observou-se que, entre as crianças de 2 meses de idade, 0 comparecimento para a consulta foi maior que entre aquelas de mais idade. Este resultado pode ser interpretado de forma positiva, visto que é no período neonatal que a criança necessita de uma maior vigilância e acompanhamento profissional, em decorrência da alta taxa de mortalidade infantil nesta etapa da vida. Não obstante, é importante que se destaque a necessidade das crianças maiores de serem acompanhadas pelo serviço com vistas ao monitoramento do crescimento e desenvolvimento psicomotor, incentivo e apoio ao aleitamento materno, imunizações e avaliação do seu estado de saúde geral.

É importante que as atividades desenvolvidas pelo serviço de puericultura não se limitem somente a este acompanhamento. Estudos têm mostrado que os serviços de puericultura são também importantes por mesclar as orientações preventivas com a troca de experiências entre os pais, com resultados positivos em termos de aquisição de conhecimentos e também na promoção das interações mãe-filh $0^{5,13,14}$.

Quanto às características das condições socioeconômicas das famílias, importantes contribuições para o entendimento do processo saúde-doença têm sido dadas pelo estudo epidemiológico ao investigar os determinantes sociais e suas influências sobre a quantidade e qualidade de saúde em grupos populacionais ${ }^{15,16}$. No presente estudo, observou-se que as crianças pertenciam a famílias cuja renda era, na sua maior parte, muito baixa, determinando precárias condições de vida. Estudo realizado com pré-escolares em outra cidade da Zona da Mata do estado de Minas Gerais mostrou resultado semelhante, sendo a renda per capta menor que $\mathrm{R} \$ 80,00^{7}$, o que nos informa sobre a necessidade de investimento e políticas de desenvolvimento local.

De maneira mais ampla, Monteiro ${ }^{16}$ também destaca que a tendência secular das condições de saúde na infância, em qualquer população, é for temente condicionada pela evolução do poder aquisitivo das famílias com crianças e pela situação da escolaridade dos membros destas. Do poder aquisitivo das famílias, dependem, por exemplo, a disponibilidade de alimentos, a qualidade da moradia e o acesso a serviços essenciais, como os de saneamento e os de assistência à saúde ${ }^{16}$.

Da escolaridade dos familiares, sobretudo da escolaridade materna, depende a utilização mais ou menos eficiente (do ponto de vista do cuidado infantil) da renda e dos serviços que estiverem ao alcance. A escolaridade materna, e dos demais familiares da criança, exerce influência também sobre as oportunidades de emprego e de salários e, nessa medida, condiciona o próprio poder aquisitivo ${ }^{16}$. No presente estudo, os pais se apresentaram com uma baixa escolaridade, o que pode estar contribuindo para uma adesão e freqüência ao serviço, bem como negativamente, em alguns indicadores de saúde.
A idade média materna foi 27,5 ( $\pm 5,45)$ anos, e observouse que a prevalência de mães na faixa de idade encontrou-se dentro da recomendada pela obstetrícia para mulheres em idade reprodutiva, o que se mostrou diferente da observada por outros autores em estudos desenvolvidos no país, em que há um predomínio de mães adolescentes ${ }^{17,18}$. Não obstante, torna-se importante que se destaque que $11,5 \%$ das mães estudadas eram adolescentes.

No presente estudo, observou-se que um maior percentual das mães que residiam em locais de fácil acesso à unidade de saúde levou seus filhos à consulta da puericultura. A acessibilidade das famílias à unidade básica de saúde é um ponto importante a ser analisado pelos gestores municipais, por ser um dos princípios do Sistema Único de Saúde ${ }^{19}$, determinando, portanto, a maior efetividade das ações e serviços sanitários.

Com relação à prestação de serviço pré-natal, observou-se uma relação positiva entre a assistência à gestante e, posteriormente, à criança, na unidade de saúde. Os dados sugerem que mães que receberam assistência pré-natal, realizando seis ou mais consultas, bem como aquelas que iniciaram precocemente o pré-natal e compareceram ao puerpério, provavelmente foram aquelas que levaram as crianças as consultas da puericultura. No presente estudo, os resultados estão em consonância com as recomendações do Ministério da Saúde ${ }^{20}$, que preconiza um mínimo de seis consultas no acompanhamento à gestante.

A situação de moradia e o saneamento foram variáveis analisadas nas famílias das crianças assistidas no serviço. Estas condições determinam não somente o conforto, mas a saúde dos indivíduos em uma sociedade, e os resultados relacionados ao número de habitantes por domicílio aproximam-se do encontrado pelo IBGE ${ }^{21}$, onde o número de 3,7 habitantes/ domicílio prevalece para o conjunto do país. Em relação às condições de saneamento, os resultados apontam para uma precariedade de saneamento básico dos domicílios, destacando-se como fator de risco a saúde da população; resultados semelhantes foram encontrados em outros estudos realizados em zona rura| ${ }^{12,23}$.

No que se refere às mães que trouxeram seus filhos ao serviço de puericultura, observou-se que as gestações a termo alcançaram uma boa prevalência. Há que se destacar que a pré-maturidade e o baixo peso ao nascer são considerados as principais causas de morbimortalidade nos primeiros anos de vida. Neste sentido, a média do peso ao nascer foi de 3.060 $( \pm 448 \mathrm{~g})$, enquanto o percentual de crianças com peso baixo ao nascer encontrou-se discretamente abaixo do valor observado em crianças brasileiras pela PNDS ${ }^{17}$. Com base nos dados obtidos, pode-se inferir que a assistência pré-natal prestada pela equipe do PSF em estudo mostra-se adequada, destacando-se também a alta prevalência de gestações a termo e o peso ao nascer acima da média.

A cobertura vacinal foi $84 \%$, e o Ministério da Saúde avalia como satisfatória uma cober tura em torno de $95 \%$, devendose, portanto, se estabelecer como pauta da ESF a necessidade de se priorizar este aspecto em seu plano de atuação.

Concernente à alimentação do lactente, o leite é considerado um alimento básico. A escolha do tipo de leite, 
especialmente nos 6 primeiros meses de vida, tem importante papel na manutenção do adequado estado nutricional e na prevenção de deficiências de nutrientes essenciais para promover o crescimento e o desenvolvimento saudável, bem como na proteção do sistema imunológico, protegendo o infante contra infecções e, além desses fatores fisiológicos mencionados, o desenvolvimento emocional por meio do fortalecimento do vínculo mãe/filho ${ }^{24,16}$. A prevalência da duração do aleitamento materno no presente estudo foi de 7,25 meses, superior àquela apresentada pela PNDS (92\%), para o país como um todo, e inferior a da OMS, que preconiza 0 alongamento do aleitamento materno até 24 meses de idade ${ }^{17}$. Ressalta-se, portanto, que este deve ser um aspecto a ser priorizado pela equipe de saúde da família em sua pauta de atuação.

Com respeito às internações hospitalares, os resultados do presente estudo apontam para uma baixa prevalência de internação, diferenciando-se dos achados de ISSLER et al. ${ }^{23}$. Estes autores mostraram que crianças oriundas de famílias vivendo em condições de pobreza são mais vulneráveis e apresentam maior número de internações hospitalares. 0 maior número de internação pode ser explicado pela situação de pobreza, que engloba problemas de ordem social, econômica e cultural das famílias, e pela inadequada cobertura e escassa resolutividade dos serviços de saúde no nível primário ${ }^{25}$. Talvez no que se refere a este ponto em específico, as atividades desenvolvidas pela equipe de saúde em nível de atenção primária mostram-se de especial importância para a promoção e prevenção de morbidades que determinam internações hospitalares no grupo infantil.

\section{Referências}

1. Ministério da Saúde(BR) Saúde da família: uma estratégia para a reorientação do modelo assistencial. Brasília(DF); 1994.

2. Senna MCM, Cohen MM. Modelo assistencial e estratégia saúde da família no nível local: análise de uma experiência. Cienc Saude Colet 2002; 3: 7.

3. Lira PIC, Lima MC, Silva GAP, Ronami SAM, Eickmann SH et al. Child health and nutrition in urban areas in the South of Pernambuco: preliminary results of a cohort study. Rev Bras Saude Mater Infant 2003; 3 (4): 463-72.

4. Lansky S, França E, Leal MC. Mortalidade perinatal e evitabilidade: revisão da literatura. Rev Saude Publica 2002; 36 (6): 759-72.

5. Blank D. A puericultura hoje: um enfoque apoiado em evidências. J Pediatr 2003; 79 (supl 1): S13-S22.

6. Accioly E, Saunders C, Lacerda EMA, et al. Nutrição em obstetrícia e pediatra. Rio de Janeiro(RJ); 2002. p. 281-88.

7. Cavalcante AAM. Consumo alimentar, perfil nutricional e de saúde de crianças no $2^{\circ}$ e $3^{\circ}$ anos de vida atendidas nos serviços públicos de saúde do município de Viçosa, Minas Gerais. [dissertação de mestrado]. Viçosa(MG): Programa de Pós-Graduação em Ciência da Nutrição/UFV; 2004.

8. Green M, Palfrey JS. Bright futures: guidelines for healthsupervision of infants, children, and adolescents. [on-line] 2002. [citado $23 \mathrm{fev}$ 2003].Arlington(USA): National Center for Education in Maternal and Child Health; 2002. Disponivel em: www.brightfutures.org/bf2/index.html.

\section{CONCLUSÃO}

Diante dos resultados deste estudo pôde-se concluir que os mesmos permitiram a obtenção de importantes informações sobre a prestação de cuidados primários em saúde para o grupo materno-infantil atendido no PSF e, sobretudo, a avaliação da assistência prestada, bem como permitiu um melhor conhecimento das condições de saúde, socioeconômicas e demográficas desta população.

0 presente estudo contribuiu também para detectar as falhas nas ações de saúde, entendê-las e considerá-las como ferramentas de motivação para promover melhoria na assistência ao grupo materno-infantil, assim como promover mudanças de atitudes e comportamentos dos profissionais envolvidos na efetividade destas ações.

Deve-se destacar que os resultados mostraram lacunas no serviço de puericultura, ressaltando-se o número insatisfatório de consultas quando relacionado ao preconizado pelo Ministério da Saúde, embora tenha sido encontrado resultado satisfatório com relação à primeira consulta anterior aos dois meses de vida.

Evidentemente, as melhorias de acesso aos serviços de saúde vão depender de um redirecionamento das políticas e programas de saúde, onde os profissionais devem se sentir motivados para desenvolver o trabalho em equipe, de forma articulada e planejada, visando alcançar a melhor assistência possível e de qualidade, para assim, promover e possibilitar mudanças positivas nos indicadores de saúde da população assistida.

9. Hoekelman RA. Child health supervision. In: Hoekelman RA, Friedman SB, Nelson NM, Seidel HM, Weitzman ML, Wilson MEH, editores. Primary

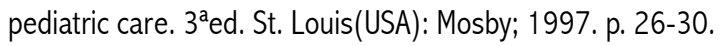

10. Druss BG, Marcus SC, Olfson M, Tanielian T, Pincus HA. Trendsin care by nonphysician clinicians in the United States. N Engl J Med 2003; 348: 130-37.

11. Dean AG, Dean JA, Coulombier D, et al. Epi info, Version 6.04a, a word processing, database, and statistics programs for public health on IBM-compatible microcomputer. Atlanta(USA): Center for Health Disease Control and Prevention; 1996.

12. World Health Organization-WHO. The optimal duration of exclusive breastfeeding. Report of an expert consultation. Geneve(SW); Mar 2001.

13. Rice RL, Slater CJ. An analysis of group versus individual child health supervision. Clin Pediatr 1997; 36: 685-89.

14. Taylor JA, Davis RL, Kemper KJ. A randomized controlled trial of group versus individual well child care for high-risk children: maternalchild interaction and developmental outcomes. Pediatrics 1997; 99: E9.

15. Tomasi E, Victoria FC. Situação sócio-econômica e condições de vida: separação de duas coortes de base populacional no sul do Brasil. Cad Saude Publica 1996; 12 (supl. 1): 15-7.

16. Monteiro CA, Freitas IC. Evolução de condicionantes socioeconômicos da saúde na infância na cidade de São Paulo:19841996. Rev Saude Publica 2000; 34 (supl 6): 83-90. 
17. Pesquisa Nacional sobre Demografia e Saúde- PNDS. Indicadores e dados básicos- Brasil 1996. Rio de Janeiro(RJ): BENFAM/IBGE/ DHS/FNUAP/UNICEF; 1997.

18. Silva DG. Fatores de risco para anemia ferropriva em lactentes do município de Viçosa, Minas Gerais. [dissertação de mestrado]. Viçosa(MG): Programa de Pós-Graduação em Ciência da Nutrição/ UFV; 2003.

19. Negri Filho A. Epidemiologia e planejamento estratégico: a experiência de Icapuí e suas lições. In: Sistemas locais de saúde em municípios de pequeno porte: a resposta de Icapuí. Fortaleza(CE); 1992. p. 27-36.

20.Ministério da Saúde.(BR). Secretaria de Políticas de Saúde. Programa de Humanização do Pré-natal e Nascimento. Brasília(DF); 2000.

21.Instituto Brasileiro de Geografia e Estatistica-IBGE. Censo demográfico. Características da população e dos domicílios: resultados do universo. Rio de Janeiro(RJ); 2000. p. 1-520.

22. Amaral LA, Nader Filho A, Rossi Junior OD, Ferreira LAF, Barros LSS. Água de consumo humano como fator de risco à saúde em

23. Issler MS, Giugliani ERJ, Kreutz GT, Meneses CF, Justo EB, Kreutz VM. Pires M. Nível de pobreza e estado de saúde das crianças: um estudo de fatores de risco em população urbana de baixo risco socioeconômico. Rev Saude Publica 1996; 30: 506-11.

24. Euclydes MP. Nutrição do lactente: base científica para uma alimentação adequada. $2^{\mathrm{a}}$ ed. Viçosa(MG); 2000.
25. BittencourtAS, Leal MC, Santos MO. Hospitalizações por diarréia infecciosa no estado do Rio de Janeiro. Cad Saude Publica 2002; 18 (3): 747-54.

\section{Sobre as Autoras}

\section{Claudete Costa De Lima}

Enfermeira, Programa Saúde da Família - Teixeiras - MG. Especialista em Nutrição e Saúde pelo Dept ${ }^{0}$ de Nutrição e Saúde da Universidade Federal de Viçosa-MG.

\section{Rosângela Minardi Mitre Cotta}

Professora Adjunta, Departamento de Nutrição e Saúde, Universidade Federal de Viçosa, Doutora em Saúde Pública pela Universidade de Valencia-Espanha.

\section{Ana Augusta Monteiro Cavalcante}

Nutricionista - Mestre em Ciências da Nutrição pelo Departamento de Nutrição e Saúde, Universidade Federal de Viçosa,

\section{Poliana Cardoso Martins}

Nutricionista - Mestranda em Ciências da Nutrição e Especialista em Nutrição e Saúde pelo Dept ${ }^{0}$ de Nutrição e Saúde da Universidade Federal de Viçosa - MG. 